EPJ Web of Conferences 16, 05002 (2011)

DOI: $10.1051 /$ epjconf/20111605002

(C) Owned by the authors, published by EDP Sciences, 2011

\title{
Numerical simulations for terrestrial planets formation
}

\author{
J. $\mathrm{Ji}^{1, \mathrm{a}}$ and N. Zhang ${ }^{1,2}$ \\ ${ }^{1}$ Purple Mountain Observatory, Chinese Academy of Sciences, Nanjing 210008, China \\ ${ }^{2}$ Graduate School of Chinese Academy of Science, Beijing 100049, China
}

\begin{abstract}
We investigate the formation of terrestrial planets in the late stage of planetary formation using two-planet model. At that time, the protostar has formed for about $3 \mathrm{Myr}$ and the gas disk has dissipated. In the model, the perturbations from Jupiter and Saturn are considered. We also consider variations of the mass of outer planet, and the initial eccentricities and inclinations of embryos and planetesimals. Our results show that, terrestrial planets are formed in $50 \mathrm{Myr}$, and the accretion rate is about $60 \%-80 \%$. In each simulation, 3-4 terrestrial planets are formed inside "Jupiter" with masses of 0.15-3.6 $M_{\oplus}$. In the 0.5-4 AU, when the eccentricities of planetesimals are excited, planetesimals are able to accrete material from wide radial direction. The plenty of water material of the terrestrial planet in the Habitable Zone may be transferred from the farther places by this mechanism. Accretion may also happen a few times between two giant planets only if the outer planet has a moderate mass and the small terrestrial planet could survive at some resonances over time scale of $10^{8} \mathrm{yr}$.
\end{abstract}

\section{INTRODUCTION}

The discovery of the extrasolar planets [1-3] around solar-type stars indeed provides substantial clues for the formation and origin of our own solar system. According to standard theory [4-6], it is generally believed that planet formation may experience such several stages: in the early stage, the dust grains condense to grow km-sized planetesimals; in the middle stage, Moon-to-Mars sized embryos are created by accretion of planetesimals. When the embryos grow up to a core of $\sim 10 M_{\oplus}$, runaway accretion may occur. With excess gases accreted onto the solid core, the embryos become more massive and eventually collapse to produce giant Jovian planets [7]. At the end of the stage, it is around that the protostar has formed for about $3 \mathrm{Myr}$, the gas disk has dissipated. A few larger bodies with low $e$ and $i$ are in crowds of planetesimals with certain eccentricities and inclinations. In the late stage, the terrestrial embryos are excited to high eccentricity orbits by mutual gravitational perturbation. Next, the orbital crossings make planets accrete stuff in wider radial area. In this sense, solid residue is either scattered out of the planetary system or accreted by the massive planet, even being captured [8] at the resonance position of the giant planets.

Chambers studied terrestrial planet formation in the late stage by numerical simulations [9] and set 150-160 Moon-to-Mars size planetary embryos in the 0.3-2.0 AU under mutual interactions by Jupiter and Saturn. The author further examined two initial mass distributions: approximately uniform masses, and a bimodal mass distribution. The results show that 2-4 planets are formed within $50 \mathrm{Myr}$, and finally survive over $200 \mathrm{Myr}$ with eventual orbits in larger eccentricities and higher inclinations. Raymond et al. also investigated the formation of terrestrial planets $[10,11]$ by simply considering Jupiter's gravitational perturbation, and the distribution of material are in 0.5-4.5 AU. Those confirm a leading hypothesis for

ae-mail: jijh@pmo.ac.cn

This is an Open Access article distributed under the terms of the Creative Commons Attribution-Noncommercial License 3.0, which permits unrestricted use, distribution, and reproduction in any noncommercial medium, provided the original work is properly cited. 
the origin of Earth's water: they may come from the material in the outer area by impacts in the late stage of planet formation. Moreover, they explored the planet formation under planetary migration of the giant [12], where super Earth may form interior to the migrating giant, and water-rich Earth-size terrestrial planet are finally found in the Habitable Zone (0.8-1.5 AU) and survive over $10^{8} \mathrm{yr}$.

In our work, we consider two-planet model, in which Jupiter and Saturn are supposed to be already formed, with two swarms of planetesimals distributed among 0.5-4.2 AU and 6.2-9.6 AU respectively. The initial eccentricities and inclinations of planetesimals are considered. We also vary the mass of Saturn to examine how the small bodies evolve. The simulations are performed on longer timescale 400 Myr with MERCURY package [13], in order to check the stability and the dynamical structure evolution of the system. In the following, we briefly summarize our numerical setup and results.

\section{NUMERICAL SETUP}

The timescale of formation of Jupiter-like planets is usually considered to be less than $10 \mathrm{Myr}$ [14], and the formation scenario of planetary embryos is relevent to their heliocentric distances and initial mass of the nebular. If we adopt the model of 1.5 MMSN (Minimum Mass Solar Nebular), the upper limit of the timescale for gas giants formation corresponds to that of embryo formation at 2.5 AU [15], which is at $3: 1$ resonance area of Jupiter. In the 2.5-4.2 AU, embryos will be cleared off by strong perturbation from Jupiter. There should be some much smaller solid residue among Jupiter and Saturn, even though the clearing effect may throw out most of the material in this area. We set embryos simply in the region 0.5-2.5 AU and planetesimals at 0.5-4.2 AU and 6.2-9.6 AU. Here, we equally set the masses of planetesimals inside and outside Jupiter, respectively. Consequently, the number distribution of the planetesimals is simply required to meet $N \propto r^{-1 / 2}$. Additionally, we remain the total number of planetesimals and embryos inside Jupiter, and the number of planetesimals outside Jupiter both equal to 200. The mass inside and outside Jupiter is equal to be $7.5 M_{\oplus}$. The eccentricities and inclinations vary in $(0-0.02)$ and $\left(0-0.05^{\circ}\right)$, respectively. The mass of Saturn in simulations $1 \mathrm{a} / 1 \mathrm{~b}, 2 \mathrm{a} / 2 \mathrm{~b}$ and $3 \mathrm{a} / 3 \mathrm{~b}$ are $0.5 M_{\oplus}, 5 M_{\oplus}, 50 M_{\oplus}$ respectively. Each simulations marked by label a (or b) is run to consider (not consider) self-gravitation of planetesimals among giants.

\section{RESULTS}

Figure 1 is a snapshot of simulation $2 \mathrm{a}$. At $0.1 \mathrm{Myr}$, it is clear that the planetesimals are excited at the $3: 2$ (3.97 AU), $2: 1$ (3.28 AU) and $3: 1$ (2.5 AU) resonance positions with Jupiter, and this is quite similar to Kirkwood gaps of the main asteroidal belt in solar system. For about $1 \mathrm{Myr}$, planetesimals and embryos are deeply intermixed and most of them are stirred to have large eccentricities. Collisions and accretions frequently emerge among planetesimals and embryos. And most of the planetary embryos are formed at about $50 \mathrm{Myr}$. The formation timescale of embryos is in accordance with that of [7]. Finally, inside Jupiter, 3 terrestrial planets are formed with masses of 0.15-3.6 $M_{\oplus}$. However, at the outer region, planetesimals are continuously scattered out of the system at $0.1 \mathrm{Myr}$. For $\sim 10 \mathrm{Myr}$, there are no survivals except at some resonances (e.g., $1: 2$ ) with Jupiter. The scattering of planetesimals cause Jupiter (Saturn) migrates inward (outward) 0.13 AU (1.19 AU) toward the sun respectively. Such migration agrees with the work of Fernandez et al. [16] Hence, $2: 5$ mean motion resonance is destroyed, then the ratios of periods for Jupiter and Saturn degenerate to $1: 3$. Therefore, the ratio of periods for Jupiter, small body and Saturn is approximate to $1: 2: 3$. In $0.5-4$ AU, when the eccentricities of planetesimals are excited, planetesimals are able to accrete stuff in wide radial direction. The plenty of water material of the terrestrial planet in the Habitable Zone may be transferred from distant region by this mechanism.

Figure 2 is shown for simulation $2 \mathrm{~b}$. It is apparent that planetesimals are excited more quickly at the $3: 2$ (3.97 AU), $2: 1$ (3.28 AU) and $3: 1$ (2.5 AU) resonance location with Jupiter, compared with Figure 1. The several characteristic timescale is same as simulation 2 a for small bodies within Jupiter. 
Research, Science and Technology of Brown Dwarfs and Exoplanets

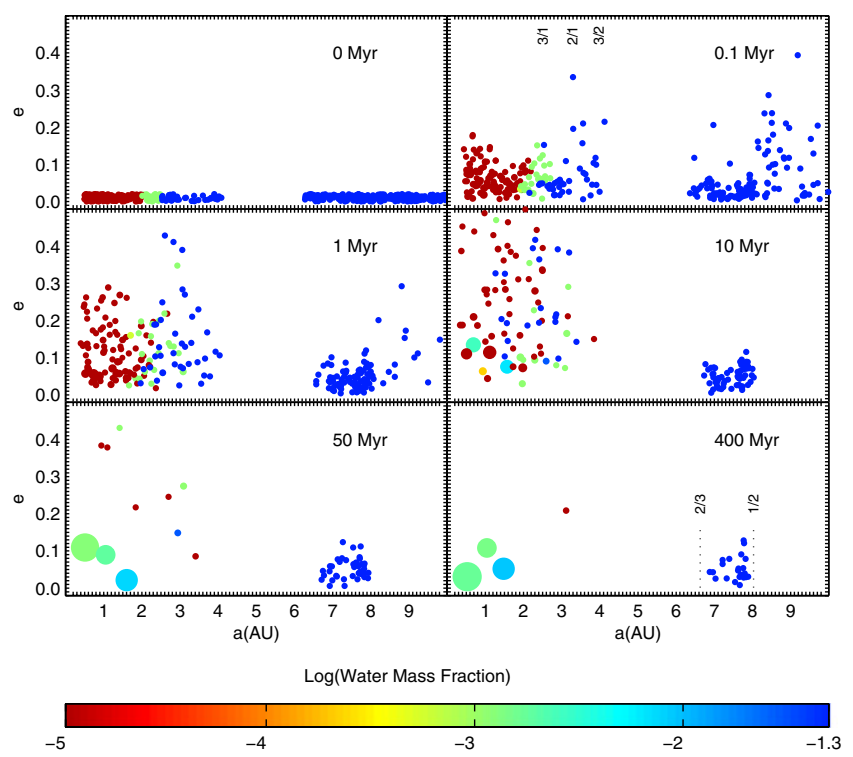

Figure 1. Snapshot of simulation 2a with $M_{\text {Saturn }}=5 M_{\oplus}$. The total mass of embryos is $2.4 M_{\oplus}$, the masses of planetesimals inside Jupiter are $0.0317 M_{\oplus}$, and those outside Jupiter are $0.0375 M_{\oplus}$. Planetesimals among Jupiter and Saturn were nonself-gravitational. Note the size of each object is relative, and the value bar is log of water mass fraction.

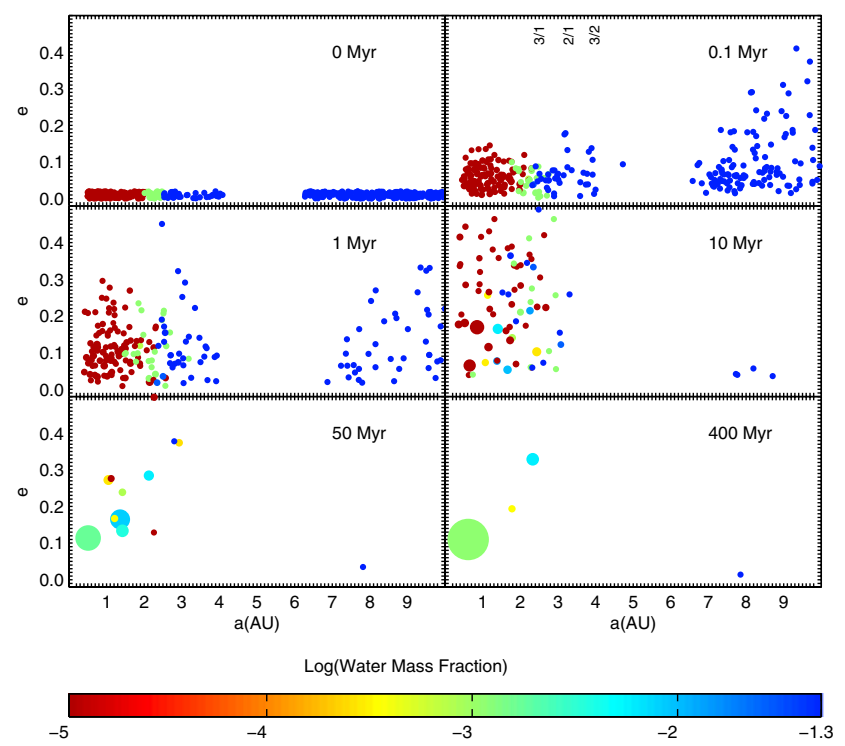

Figure 2. Snapshot for similuation $2 b$.

Here 4 planets are formed. In this run, we consider self-gravitation among the outer planetesimals. Several planetesimals do survive over $400 \mathrm{Myr}$ about $7-8 \mathrm{AU}$ in the area of $2: 3$ (6.63 AU) and $1: 2$ (8.03 AU) resonances with Jupiter. The detailed results for whole simulations that the reader may refer to [17]. 


\section{EPJ Web of Conferences}

\section{SUMMARY}

We simulate terrestrial planet formation by using two-planet model. In the simulations, the variations of the mass of outer planet, the initial eccentricities and inclinations of embryos and planetesimals are also considered. The results show that, during the terrestrial planet formation, planets can accrete material from various areas inside Jupiter. In the regime 0.5-4.2 AU, accretion rate of terrestrial planets is 60\%$80 \%$, which indicates that $20 \%-40 \%$ initial mass is removed over the evolution. The planetesimals will improve efficiency of accretion rate for certain initial eccentricities and inclinations. Such adoption may cause newly-born terrestrial planets to bear lower orbital eccentricities. It also indicates that in the planet formation that water-rich terrestrial planet may be formed in the Habitable Zone. Most of the planetesimals among Jupiter and Saturn are scattered out of the system, and such migration induced by scattering [16] or long-term orbital evolution can make smaller bodies capture at some mean motion resonance location. Accretion could also happen a few times between two planets if the outer planet owns a moderate mass, and a small terrestrial planet could survive at some resonances over time scale of $10^{8} \mathrm{yr}$. The outcomes further reveal that the outer planet has little effect on dynamical architecture inside Jupiter.

This work is financially supported by the National Natural Science Foundations of China (Grants 10973044, 10833001, 10573040, 10673006), and the Foundation of Minor Planets of Purple Mountain Observatory.

\section{References}

[1] Mayor, M., \& Queloz, D. Nature 378, 355 (1995)

[2] Lee, M. H., \& Peale, S. J., ApJ 567, 596 (2002)

[3] Ji, J. H., et al., ApJ 585, L139 (2003)

[4] Safronov, V.S., Evolution of the Protoplanetary Cloud and Formation of the Earth and the Planets (Moscow, Nauka, 1969)

[5] Wetherill, G. W., Ann. Rev. Earth Planet Sci. 18, 205 (1990)

[6] Lissauer, J.J., ARAA 31, 129 (1993)

[7] Ida, S., \& Lin, D. N. C., ApJ 604, 388 (2004)

[8] Nagasawa, M., \& Ida, S., AJ 120, 3311 (2000)

[9] Chambers, J. E., Icarus 152, 205 (2001)

[10] Raymond, S. N., Quinn, T., \& Lunine, J. I., Icarus 168, 1 (2004)

[11] Raymond, S. N., Quinn, T., \& Lunine, J. I., Icarus 183, 265 (2006)

[12] Raymond, S. N., Mandell, A. M., \& Sigurdsson, S., Science 313, 1413 (2006)

[13] Chambers, J. E., MNRAS 304, 793 (1999)

[14] Briceño, C. et al., Science 291, 93 (2001)

[15] Kokubo, E., \& Ida, S., ApJ 581, 666 (2002)

[16] Fernandez, J. A., \& Ip, W. H., Icarus 58, 109 (1984)

[17] Zhang N., Ji J., Science in China Series G 52(5), 794 (2009) 